DOI: 10.12957/demetra.2015.16726

\title{
Composição química da laranja kinkan e de frutas cítricas
}

\section{Chemical composition of kinkan orange and citrus fruits}

Ariane Barros Diniz

Dirce Ribeiro de Oliveiral

1 Universidade Federal de Minas Gerais, Escola de Enfermagem, Departamento de Nutrição. Belo Horizonte-MG, Brasil.

Correspondência / Correspondence

E-mail: dirceribeiroliveira@gmail.com

\section{Resumo}

A laranja Kinkan (Fortunella margarita), conhecida popularmente como "laranja de ouro", é originária da Ásia e pertence ao gênero Fortunella. Possui algumas características das frutas cítricas, que são as mais consumidas pela população brasileira. Objetivo: O presente estudo teve como objetivo avaliar a caracterização química da laranja kinkan, comparando os resultados obtidos com os dados publicados na análise de algumas frutas cítricas de grande significado comercial no Brasil, como laranja serra-d'água, laranja pera-rio, tangerina poncã e limão taiti. Metodologia: Foram avaliados $\mathrm{pH}$, sólidos solúveis, acidez titulável, teor de vitamina $\mathrm{C}$, fenólicos totais e capacidade antioxidante. Todos os testes foram realizados em triplicata, sendo os dados comparados pelo teste de Tukey $(\mathrm{p}<0,05)$. Resultados: A laranja kinkan apresentou o maior teor de sólidos solúveis (SS) e baixa acidez titulável (AT); consequentemente, alta relação SS/AT, elevado teor de vitamina $\mathrm{C}$ e o maior teor de fenólicos totais em relação às demais frutas analisadas. Conclusão: A laranja kinkan possui características químicas semelhantes às outras frutas cítricas, como $\mathrm{pH}$, acidez titulável e teor de vitamina C, apresenta excelente palatabilidade e pode ser considerada como fonte de fenólicos totais e de vitamina C na alimentação.

Palavras-chave: Citrus. Vitamina C. Polifenóis. Capacidade Antioxidante. 


\section{Abstract}

Orange Kinkan (Fortunella margarita), popularly known as "golden orange", is originally from Asia and belongs to the genus Fortunella. Kinkan orange has some characteristics of citrus fruits, which are the most consumed fruits by the Brazilian population. Objective: The study aimed to evaluate the chemical characterization of kinkan orange by comparing the results obtained with the published data on the analysis of some citrus fruits of great commercial interest in Brazil, such as serra-d'água orange, pera-rio orange, poncã tangerine and tahiti lemon. Methodology: We evaluated $\mathrm{pH}$, soluble solids, titratable acidity, content of vitamin $\mathrm{C}$, total phenolics and antioxidant capacity. All tests were performed in triplicate and data were compared by Tukey test $(\mathrm{p}<0,05)$. Results: Kinkan orange had the highest content of soluble solids (SS) and low titratable acidity (TA), consequently high SS / TA ratio, high vitamin C content and the highest content of total phenolics compared to other fruits. Conclusion: Kinkan orange has chemical characteristics similar to other citrus fruits such as $\mathrm{pH}$, titratable acidity and content of vitamin $\mathrm{C}$, has excellent palatability and can be considered as a source of total phenolic and vitamin $\mathrm{C}$ in the diet.

Key words: Citrus. Vitamin C. Polyphenols. Antioxidant Capacity.

\section{Introdução}

As plantas cítricas do gênero Citrus e os Kunquats do gênero Fortunella pertencem à família Rutaceae, são nativas do sudeste asiático, com ramos filogenéticos que se estendem do centro da China ao Japão, e do leste da Índia a Nova Guiné, Austrália e África Tropical. ${ }^{1}$

A laranja kinkan (Fortunella margarita), conhecida popularmente como "laranja de ouro", é originária da Ásia, apresenta árvore de pequeno porte, floresce na primavera e verão e seus frutos aparecem principalmente no outono. ${ }^{1}$ É uma planta que pode ser cultivada para fins ornamentais em jardins e interiores. ${ }^{2}$

Apesar de pertencer ao gênero Fortunella, a laranja kinkan possui algumas características das frutas cítricas, como aroma e sabor, porém contém menor quantidade de gomos e casca mais fácil de digerir. ${ }^{2}$ Pode ser consumida crua e inteira, com exceção das sementes; sua casca é macia, doce e tem aroma típico devido principalmente à presença de flavonoides e terpenoides. Pode ser usada no preparo de caldas, molhos ou compotas, adicionada a saladas de frutas, preservadas inteiras em xarope de açúcar ou usada como decoração. ${ }^{3,4}$ 
Os ácidos orgânicos, açúcares e compostos fenólicos estão entre os principais compostos presentes nas frutas cítricas, sendo importantes fatores de sua qualidade, uma vez que os compostos fenólicos conferem propriedades antioxidantes e os açúcares, os atributos de sabor. ${ }^{5}$ Os ácidos cítrico e málico são os principais ácidos orgânicos componentes das frutas cítricas. Os sólidos solúveis e a doçura do suco estão diretamente ligados à quantidade de açúcar na fruta. ${ }^{6}$

Quanto às vitaminas presentes nas frutas cítricas, a vitamina C é predominante, ${ }^{7}$ sendo encontrada em valores entre 50 a 100mg de ácido ascórbico/100g. ${ }^{8}$ Destacam-se ainda vitamina A e vitaminas do complexo B, além de sais minerais, principalmente cálcio, potássio, sódio, fósforo e ferro. ${ }^{9}$

As frutas cítricas são as mais consumidas pela população brasileira. Há relatos de consumo anual per capita de 5,59Kg de laranja, $0,565 \mathrm{Kg}$ de limão e $1,17 \mathrm{Kg}$ de tangerina. ${ }^{10}$ Não há dados sobre o consumo da laranja kinkan, mas sabe-se que no Brasil é amplamente cultivada, sobretudo na Região Sudeste. ${ }^{11}$

Utilizando-se a Tabela Brasileira de Composição de Alimentos, ${ }^{12}$ é possível obter informações sobre a composição química de diversas frutas cítricas, porém não existem dados científicos que apresentem a composição química da laranja kinkan. Desta forma, o presente estudo teve como objetivo avaliar a caracterização química da laranja kinkan (Fortunella margarita), comparando os resultados obtidos com os dados publicados na análise de algumas frutas cítricas de grande significado comercial no Brasil, como laranja serra-d'água, laranja pera-rio, tangerina poncã e limão taiti.

\section{Materiais e métodos}

\section{Amostra}

As frutas utilizadas para realização das análises foram: laranja kinkan, laranja serra-d'água, laranja pera-rio, tangerina poncã e limão taiti, in natura. O experimento foi realizado entre junho e novembro de 2011, e todas as frutas utilizadas foram provenientes do Mercado Central de Belo Horizonte (Belo Horizonte-MG).

\section{Caracterização das amostras}

Foram utilizadas variedades de tamanho médio, com peso médio de $50 \mathrm{~g}$ de laranja kinkan, $120 \mathrm{~g}$ de laranja serra-d'água, 124g laranja pera-rio pêra, $154 \mathrm{~g}$ de tangerina poncã e $100 \mathrm{~g}$ de limão taiti. Para a realização das análises, foi utilizada a laranja kinkan inteira (exceto sementes) e o suco das demais frutas. A amostragem foi realizada ao acaso e foram utilizadas amostras de 
tamanho médio de cada variedade. O suco de cada amostra foi extraído manualmente e depois homogeneizado para a realização das análises. ${ }^{13}$

As análises físico-químicas foram realizadas nas amostras e constaram das seguintes variáveis: pH, determinado em PHmetro digital marca Digimed, modelo D.M. - 20, sólidos solúveis totais ( ${ }^{\circ}$ Brix), analisado em refratômetro marca Baush \& Lomb Optical Company, modelo ABBE-3L; acidez total titulável (g de ácido cítrico/100ml de suco), determinada por titulometria com solução de hidróxido de sódio $0,1 \mathrm{~N}$ e fenolftaleína como indicador. ${ }^{13,14}$

\section{Determinação do teor de vitamina $\mathrm{C}$}

A determinação de vitamina $\mathrm{C}$ foi realizada segundo Yurena et al. ${ }^{7}$ As amostras foram congeladas a $-70^{\circ} \mathrm{C}$ até a realização das análises. Tais amostras foram trituradas em mixer. Pesou-se $0,5 \mathrm{~g}$ da amostra e misturou-se com 2,5ml da solução ácida extratora (3\% de ácido metafosfórico e $8 \%$ de ácido acético). A mistura foi homogeneizada a $18.000 \mathrm{~g}$ (no gelo e no escuro), durante um minuto e depois centrifugada a $9.000 \mathrm{~g}$ (refrigerado a $4^{\circ} \mathrm{C}$ ) durante 20 minutos. Este procedimento foi repetido duas vezes e os dois sobrenadantes resultantes foram misturados. Todas as análises foram realizadas em triplicata. Após repetição dos procedimentos e a mistura dos sobrenadantes, a amostra foi titulada com o reagente de Tillmans. ${ }^{13}$ Várias precauções foram tomadas, a fim de realizar todas as operações sob reduzida luz e a $4^{\circ} \mathrm{C}$ de temperatura. Além disso, a fim de estabilizar a vitamina C na solução ácida extratora, foi adicionado 0,099g de 2,6-tertibutilhidroxitolueno (BHT) a $1 \mathrm{mM}^{7}$.

\section{Fenólicos totais}

O teor de fenólicos totais foi determinado através do método de Folin-Ciocalteu. ${ }^{15}$ Foi feita uma curva-padrão de ácido gálico nas concentrações de 0 a $0,5 \mathrm{mg} / \mathrm{ml}$. Foram adicionados $50 \mu \mathrm{l}$ de amostra pré-homogeneizada em $350 \mu \mathrm{L}$ de metanol, em seguida adicionaram-se $250 \mu \mathrm{l}$ do reagente de Folin-Ciocalteu. Acrescentaram-se $3 \mathrm{ml}$ de água e $1 \mathrm{ml}$ de carbonato de sódio, e a solução foi agitada em vórtex, sendo a mistura deixada em repouso por uma hora em ambiente protegido da luz. A absorbância das amostras foi medida a 750nm. Os resultados foram expressos em miligramas de ácido gálico. ${ }^{15}$

\section{Capacidade antioxidante}

A atividade antioxidante foi determinada através do método de atividade sequestradora de radical, utilizando o radical 2,2-difenil-1-picrilhidrazil (DPPH), adaptado. ${ }^{16}$ As amostras foram diluídas 
em etanol (0,8 g fruta/25 mL de etanol). Em um tubo de ensaio, $500 \mu \mathrm{l}$ da amostra diluída foram misturados com $300 \mu$ l de solução de DPPH $(0,5 \mathrm{mM})$ e $3 \mathrm{ml}$ de etanol. As amostras foram deixadas em repouso no escuro e à temperatura ambiente por 45 minutos. O controle foi preparado de acordo com o procedimento acima, porém sem a adição da amostra. A absorbância foi medida a 517nm.

\section{Delineamento estatístico}

Todas as análises foram realizadas em triplicata. O programa estatístico utilizado foi o GraphPad Prism 5.0. Foi feita análise de variância com teste post hoc de Tukey $(p<0,05)$, e para verificar a correlação entre varáveis, foi realizada a Correlação de Pearson.

\section{Resultados}

A tabela 1 apresenta os resultados referentes aos valores de $\mathrm{pH}$, sólidos solúveis, acidez titulável e relação sólidos solúveis/acidez titulável (SS/AT) das frutas analisadas. De acordo com as análises, a laranja kinkan apresentou maior teor de sólidos solúveis em relação às demais frutas, elevada relação SS/AT em relação à laranja pera-rio, tangerina poncã e limão taiti, e valores intermediários de pH e de acidez titulável.

Tabela 1. Valores de pH, sólidos solúveis, acidez titulável e relação SS/AT das frutas analisadas. Belo Horizonte-MG, 2011.

\begin{tabular}{lcccc}
\hline & & $\begin{array}{c}\text { Sólidos } \\
\text { solúveis }\end{array}$ & Acidez titulável \\
\multicolumn{1}{c}{ Amostra } & $\mathrm{pH}$ & (ónrix) & $\begin{array}{c}\text { (g ácido } \\
\text { cítrico/100 ml de }\end{array}$ & SS/AT \\
& & suco $)$ & \\
\hline Laranja kinkan & $4,22 \pm 0,03^{\mathrm{b}}$ & $21,1 \pm 0,10^{\mathrm{a}}$ & $1,14 \pm 0,16^{\mathrm{b}}$ & $18,89 \pm 2,74^{\mathrm{b}}$ \\
Laranja serra d'água & $5,55 \pm 0,04^{\mathrm{a}}$ & $15,2 \pm 0,20^{\mathrm{b}}$ & $0,82 \pm 0,50^{\mathrm{b}}$ & $29,27 \pm 17,60^{\mathrm{a}}$ \\
Laranja pera-rio & $4,04 \pm 0,02^{\mathrm{c}}$ & $9,40 \pm 0,40^{\mathrm{c}}$ & $5,58 \pm 0,98^{\mathrm{a}, \mathrm{b}}$ & $1,73 \pm 0,23^{\mathrm{d}}$ \\
Tangerina poncã & $3,55 \pm 0,01^{\mathrm{d}}$ & $14,4 \pm 0,40^{\mathrm{b}}$ & $5,39 \pm 0,11^{\mathrm{a}, \mathrm{b}}$ & $2,67 \pm 0,13^{\mathrm{c}}$ \\
Limão tahiti & $2,56 \pm 0,02^{\mathrm{e}}$ & $8,35 \pm 0,35^{\mathrm{c}}$ & $9,80 \pm 1,70^{\mathrm{a}}$ & $0,87 \pm 0,12^{\mathrm{e}}$ \\
\hline
\end{tabular}

Média \pm erro padrão de duas repetições.

Letras diferentes nas colunas significam diferença ao nível de significância p<0,05. 
A tabela 2 apresenta os resultados referentes ao teor de vitamina $\mathrm{C}$, fenólicos totais e capacidade antioxidante das frutas analisadas. Os resultados demonstram que a laranja kinkan apresentou elevado teor de vitamina C e o maior teor de fenólicos totais, quando comparada às demais frutas. A capacidade antioxidante foi semelhante à encontrada para laranja serra-d'água, tangerina poncã e limão taiti.

Tabela 2. Teor de vitamina C, fenólicos totais e capacidade antioxidante das frutas analisadas. Belo Horizonte-MG, 2011.

\begin{tabular}{|c|c|c|c|}
\hline Amostra & $\begin{array}{c}\text { Vitamina } \mathrm{C} \\
\text { (mg ácido } \\
\text { ascórbico/100 ml } \\
\text { de suco) }\end{array}$ & $\begin{array}{l}\text { Fenólicos Totais } \\
\text { (mg ácido gálico/g } \\
\text { de fruta) }\end{array}$ & $\begin{array}{c}\text { Capacidade } \\
\text { Antioxidante } \\
(\%)\end{array}$ \\
\hline Laranja kinkan & $86,45 \pm 6,65^{\mathrm{a}}$ & $0,09 \pm 0,0010^{\mathrm{a}}$ & $52,99 \pm 8,83^{\mathrm{b}, \mathrm{c}}$ \\
\hline Laranja serra d'água & $63,18 \pm 3,33^{\mathrm{a}, \mathrm{b}}$ & $0,04 \pm 0,0002^{c}$ & $55,68 \pm 6,15^{\mathrm{b}, \mathrm{c}}$ \\
\hline Laranja pera-rio & $53,20 \pm 6,65^{\mathrm{b}}$ & $0,05 \pm 0,0004^{\mathrm{b}}$ & $119,08 \pm 16,56^{\mathrm{a}}$ \\
\hline Tangerina poncã & $73,15 \pm 0,00^{\mathrm{a}, \mathrm{b}}$ & $0,03 \pm 0,0008^{\mathrm{d}}$ & $31,70 \pm 3,31^{c}$ \\
\hline Limão taiti & $56,52 \pm 3,33^{\mathrm{b}}$ & $0,05 \pm 0,0016^{\mathrm{b}}$ & $86,28 \pm 3,00^{\mathrm{a}, \mathrm{b}}$ \\
\hline
\end{tabular}

Média \pm erro padrão de duas repetições.

Letras diferentes nas colunas significam diferença ao nível de significância $\mathrm{p}<0,05$

A tabela 3 apresenta a correlação entre as variáveis pH, acidez titulável e sólidos solúveis das frutas. Verificou-se forte correlação negativa entre as variáveis pH e acidez titulável e entre acidez titulável e teor de sólidos solúveis.

Tabela 3. Correlação entre as variáveis "pH", "acidez titulável" e "teor de sólidos solúveis" das frutas analisadas. Belo Horizonte-MG, 2011.

\begin{tabular}{llc}
\hline & & Correlação de Pearson \\
\hline $\mathrm{pH}$ & Acidez titulável & $-0,896294932^{*}$ \\
$\mathrm{pH}$ & Sólidos solúveis & $0,502650643^{\mathrm{ns}}$ \\
Acidez titulável & Sólidos solúveis & $-0,828230191^{*}$ \\
\hline
\end{tabular}

* nível de significância $\mathrm{p}<0,05$. ns= não significativo 
A tabela 4 apresenta a correlação entre as variáveis vitamina $\mathrm{C}$, fenólicos totais e capacidade antioxidante. Houve moderada correlação negativa entre as variáveis capacidade antioxidante e teor de vitamina $\mathrm{C}$.

Tabela 4. Correlação entre as variáveis "capacidade antioxidante", "vitamina C" e "fenólicos totais" das frutas analisadas. Belo Horizonte-MG, 2011.

\begin{tabular}{lcc}
\hline & & Correlação de Pearson \\
\hline Capacidade Antioxidante & Vitamina C & $-0,740596^{*}$ \\
Capacidade Antioxidante & Fenólicos totais & $0,084740^{\mathrm{ns}}$ \\
\hline
\end{tabular}

* nível de significância $\mathrm{p}<0,05$. ns= não significativo

\section{Discussão}

A laranja kinkan apresentou valores intermediários de $\mathrm{pH}(4,22 \pm 0,03)$ dentre as frutas analisadas no estudo e valores de $\mathrm{pH}$ determinados dentro do intervalo de 3,20 $\pm 0,01$ a 5,43 \pm 0,06, conforme relatado por Couto \& Canniatti-Brazaca, ${ }^{16}$ após analisarem outras variedades de laranjas. Valores mais altos de $\mathrm{pH}$ são preferidos para o consumo in natura, ${ }^{17}$ sugerindo que a laranja kinkan é uma fruta indicada para o consumo in natura.

A determinação da acidez titulável é usada para demostrar o grau de acidez da solução, ou seja, a quantidade de ácido cítrico presente em $100 \mathrm{ml}$ de solução. ${ }^{16}$ A laranja kinkan apresentou teor de acidez tutulável semelhante ao encontrado para algumas frutas analisadas $(1,14 \mathrm{~g}$ ácido cítrico/100ml de suco $\pm 0,16$ ), indicando sua semelhança química com estas frutas citricas.

O teor de sólidos solúveis indica a doçura da fruta em quantidade diretamente proporcional, ou seja, quanto maior o teor de sólidos solúveis, maior a doçura do fruto. ${ }^{6}$ De acordo com os resultados encontrados, a laranja kinkan apresentou o maior teor de sólidos solúveis $(21,1$ BBrix $\pm 0,10)$ e, consequentemente, maior grau de doçura em relação às outras frutas avaliadas no estudo.

Um dos parâmetros mais importantes para informar sobre a palatabilidade dos frutos é a relação entre o teor de sólidos solúveis e a acidez titulável (SS/AT). Na maioria das vezes, os consumidores brasileiros preferem elevada relação SS/AT. ${ }^{17}$ De acordo com os resultados encontrados, a laranja kinkan possui essa elevada relação SS/AT $(18,89 \pm 2,74)$, indicando que a fruta é de boa palatabilidade.

A vitamina $\mathrm{C}$ é a principal vitamina encontrada nas frutas cítricas, podendo ser considerada uma característica desses alimentos. ${ }^{9}$ É de conhecimento que esta vitamina participa de processos 
celulares de oxirredução, previne o escorbuto, é importante na biossíntese das catecolaminas, participa na defesa do organismo contra infecções, é fundamental na integridade das paredes dos vasos sanguíneos, além de ser indispensável para a formação das fibras colágenas. No entanto, não é sintetizada pelo organismo, o que torna seu consumo essencial para a saúde humana. ${ }^{18}$ Nas frutas cítricas, o teor de vitamina C geralmente se encontra em valores entre 50 a $100 \mathrm{mg} / 100 \mathrm{ml}$ de suco. ${ }^{8}$ De acordo com os resultados encontrados, a laranja kinkan apresentou elevado teor de vitamina C (86,45mg/100g), indicando que a mesma apresenta esta característica comum às frutas cítricas.

Compostos fenólicos são metabólitos secundários presentes naturalmente em frutas e hortaliças, e na maioria das vezes sua importância está relacionada à prevenção de algumas doenças. Sua funcionalidade baseia-se sobretudo em sua ação sequestradora de radicais livres, que torna possível diminuir a incidência de patologias como aterosclerose, cataratas, câncer e inflamações crônicas. ${ }^{19}$ A laranja kinkan apresentou elevado teor de fenólicos totais $(0,09 \pm 0,0010 \mathrm{mg}$ ácido gálico/g de fruta), quando comparada às demais frutas analisadas, o que permite dizer que esta fruta pode ser utilizada na alimentação como forma de prevenção de algumas patologias, dentro do contexto de uma alimentação saudável.

Diversos estudos têm apontado a vitamina C como um dos principais antioxidantes das frutas $;,{ }^{7,20}$ no entanto, esta vitamina contribui com apenas $0,4 \%$ da capacidade antioxidante. ${ }^{21}$ Por outro lado, corroborando o achado no presente estudo, Couto \& Canniatti-Brazaca ${ }^{16}$ não encontraram associação entre o teor de vitamina C e a capacidade antioxidante das frutas analisadas. Este achado sugere que a maior capacidade antioxidante da laranja kinkan pode ser justificada por outros compostos bioativos presentes. ${ }^{21}$ Da mesma forma, no presente estudo, a capacidade antioxidante não foi associada com o teor de fenólicos totais, sugerindo que outros compostos não avaliados, como antocianinas e carotenoides, possam ser os responsaveis pela capacidade antioxidante das frutas. ${ }^{22}$.

\section{Conclusões}

A laranja kinkan possui características químicas semelhantes às encontradas em frutas cítricas, como pH, acidez titulável e teor de vitamina C. Apresenta boa palatabilidade e pode ser considerada como fonte de fenólicos totais e de vitamina C na alimentação.

\section{Agradecimentos}

A toda equipe do Laboratório de Amebíase do Instituto de Ciências Biológicas da Universidade Federal de Minas Gerais, e a Jamil Silvano, técnico do Laboratório de Bioquímica de Proteínas do Departamento de Bioquímica do ICB/UFMG. 


\section{Referências}

1. Donadio LC, Mourão-Filho FAA, Moreira CS. Centros de origem, distribuição geográfica das plantas cítricas e histórico na citricultura no Brasil. Citros 2005; p. 2-18.

2. Rocha JS, Soares Filho WS. Desenvolvimento de variedades-copa híbridas de citros: plantas ornamentais. In: Anais da Jornada Científica Embrapa Mandioca e Fruticultura Tropical, 3., 2009, Cruz das Almas. Cruz das Almas: Embrapa Mandioca e Fruticultura Tropical, 2009. Disponível em: https://www.embrapa.br/busca-de-publicacoes/-/publicacao/656450/desenvolvimento-devariedades-copa-hibridas-de-citros-plantas-ornamentais.

3. Kawaii S, Tomono Y, Katase E, Ogawa K, Yano M. Quantitation of flavonoid constituents in Citrus fruits. Journal of Agricultural and Food Chemistry 1999; 47(9): 3565-3571.

4. Schirra M, Palma A, D’Aquino S, Angioni A, Minello EV, Melis M, et al. Influence of postharvest hot water treatment on nutritional and functional properties of Kumquat (Fortunella japonica Lour. Swingle Cv.Ovale). Journal of Agricultural and Food Chemistry 2008; 56(2):455-460.

5. Angelo PM, Jorge N. Compostos fenólicos em alimentos: uma breve revisão/Phenolic compounds in foods: a brief review. Revista do Instituto Adolfo Lutz 2007; 66(1):1-9.

6. Kelebek H, Selli S. Determination of volatile, phenolic, organic acid and sugar components in a Turkish cv. Dortyol (Citrus sinensis L. Osbeck) orange juice. Journal of the Science of Food and Agriculture 2011; 91(10): 1855-1862.

7. Yurena H, Lobo MG, González M. Determination of vitamin C in tropical fruits: A comparative evaluation of methods. Food Chemistry 2006; 96: 654-664.

8. Andrade RSG, Diniz MCT, Neves EA, Nóbrega JA. Determinação e distribuição de ácido ascórbico em três frutos tropicais. Eclética Química 2002; 27(spe):393-401.

9. Mattos Junior D, Negri JD, Figueiredo JO, Pompeu Junior J. Citros: principais informações e recomendações de cultivo. Campinas: Instituto Agronômico de Campinas; 2005. p. 19-36. Boletim Técnico 200.

10. Silveira FG, Servo LM, Menezes T, Piola SF. Gasto e consumo das famílias brasileiras contemporâneas. v. 2. Brasília: IPEA; 2007. 552 p.

11. Corrêa MP. Dicionário das plantas úteis do Brasil e das exóticas cultivadas. Rio de Janeiro: Instituto Brasileiro de Desenvolvimento Florestal; 1984. 6 v.

12. Universidade Estadual de Campinas. Tabela Brasileira de Composição de Alimentos (TACO). $2^{a}$ ed. Campinas: Ed. NEPA-UNICAMP; 2006. 113 p.

13. Instituto Adolfo Lutz. Normas analíticas do Instituto Adolfo Lutz: métodos químicos e físicos para análise de alimentos. $3^{\mathrm{a}}$ ed. São Paulo: Ed. IMESP; 1985. p. 571-685.

14. Silva PT, Fialho E, Lopes MLM, Valente-Mesquita VL. Sucos de laranja industrializados e preparados sólidos para refrescos: estabilidade química e físico-química. Ciência e Tecnologia de Alimentos 2005; 25(3):597-602. 
15. Association of Official Analytical Chemistry. Official methods of analysis of AOAC international. $18^{a}$ ed. Maryland: AOAC International; 2005. 1501 p.

16. Couto MAL, Canniatti-Brazaca SG. Quantificação de vitamina C e capacidade antioxidante de variedades cítricas. Ciência e Tecnologia de Alimentos 2010; 30(supl. 1):15-19.

17. Hansen DS, Silva AS, Fonseca AAO, Hansen OAS, França NO. Caracterização química de frutos de jenipapeiros nativos do Recôncavo Baiano visando ao consumo natural e industrialização. Revista Brasileira de Fruticultura 2008; 30(4):964-969.

18. Manela-Azulay M, Mandarim-de-Lacerda CA, Perez MA, Filgueira AL, Cuzzi T. Vitamina C. Anais Brasileiros de Dermatologia 2003; 78(3):265-274.

19. Araújo PFDE, Rodrigues RS, Machado AR, Santos VS, Silva JA. Influência do congelamento sobre as características físico-químicas e o potencial antioxidante de néctar de amora-preta. Boletim do Centro de Pesquisa de Processamento de Alimentos 2009; 27(2):199-206.

20. Segantini DM, Leonel S, Lima GPP, Costa SM, Ramos ARP. Caracterização da polpa de pêssegos produzidos em São Manuel-SP. Ciência Rural 2012; 42(1):52-57.

21. Sun J, Chu YF, Wu X, Liu RH. Antioxidant and antiproliferative activities of common fruits. Journal of Agricultural and Food Chemistry 2002; 50(25):7449-7454.

22. Santos GM, Maia GA, Sousa PHM, Costa JMC, Figueiredo RW, Prado GM. Correlação entre atividade antioxidante e compostos bioativos de polpas comerciais de açaí (Euterpe oleracea Mart). Archivos Latinoamericanos de Nutricion 2008; 58(2): 187-192.

Recebido: $11 / 6 / 2015$

Revisado: 29/9/2015

Aprovado: 18/10/2105 